\title{
Une crise financière au Japon qui n'empêche pas les cerisiers de fleurir
}

(en annexe, un texte compémentaire de Jean-Robert Pitte)

\section{Sylvie Guichard-Anguis}

\section{(2) OpenEdition \\ Journals}

Édition électronique

URL : http://journals.openedition.org/echogeo/11198

DOI : 10.4000/echogeo. 11198

ISSN : 1963-1197

Éditeur

Pôle de recherche pour l'organisation et la diffusion de l'information géographique (CNRS UMR 8586)

Référence électronique

Sylvie Guichard-Anguis, "Une crise financière au Japon qui n'empêche pas les cerisiers de fleurir », EchoGéo [En ligne], Sur le Vif, mis en ligne le 04 juin 2009, consulté le 03 mai 2019. URL : http://

journals.openedition.org/echogeo/11198; DOI : 10.4000/echogeo.11198

Ce document a été généré automatiquement le 3 mai 2019.

\section{(c) (†) $\odot$}

EchoGéo est mis à disposition selon les termes de la licence Creative Commons Attribution - Pas

d'Utilisation Commerciale - Pas de Modification 4.0 International 


\title{
Une crise financière au Japon qui n'empêche pas les cerisiers de fleurir
}

(en annexe, un texte compémentaire de Jean-Robert Pitte)

\author{
Sylvie Guichard-Anguis
}

\section{Une crise vécue dans l'archipel japonais comme l'arrivée d'une catastrophe naturelle}

1 Depuis quelques mois les grands titres des quotidiens japonais comme les éditions spéciales des chaînes de télévision ne cessent de souligner l'ampleur de la crise telle un tsunami venu de l'extérieur, qui frappe l'archipel, dressant un tableau de plus en plus sombre de son avenir. Les secteurs des biens de consommation durable sont les plus touchés à la suite de l'effondrement de leurs deux principaux marchés, la Chine et les Etats-Unis. Ce sont les fleurons de l'industrie japonaise, les gagnants (kachi-gumi) de ces dernières décennies qui présentent les signes les plus inquiétants, hormis Nintendo qui affiche des bénéfices presque insolents face à la conjoncture. Les plans sociaux se succèdent à un rythme soutenu dans tout le pays et il n'y a pas de jours sans que l'un des grands noms de l'industrie électronique n'annonce la fermeture d'une ou plusieurs usines, la réduction du temps de travail hebdomadaire etc. Si dans une première étape son partage entre les employés permet de conserver les effectifs, des solutions plus dramatiques commencent à faire leur apparition et déjà certaines de ces sociétés connues dans le monde entier envisagent de licencier une part de leurs effectifs. Or se séparer d'une partie de la main d'œuvre dans un pays qui du point de vue démographique en manque et reste très réticent à la venue de travailleurs immigrés, reste une décision très lourde de conséquences pour l'avenir. Cette question donne lieu à de grands débats dans les médias, qui interrogent la responsabilité des entreprises vis à vis de leur personnel, et les limites de la protection de l'emploi. 
2 Il va sans dire que les conséquences de tels choix se révèlent dramatiques dans de nombreuses collectivités locales. Quelques unes d'entre elles réagissent en se portant acquéreurs d'une faible part des stocks accumulés (voitures, téléviseurs etc.) provoquant de vives critiques sur l'usage du fruit des impôts locaux. Les employés de certaines usines devenus inactifs sont "prêtés " pour effectuer des travaux d'intérêt général. D’autres complètent leur salaire amputé des heures non effectuées par des emplois secondaires, au risque de se surmener. Des trésors d'ingéniosité sont déployés par des chefs d'entreprises pour occuper leur effectif devenu pléthorique. Ainsi des reconversions tout à fait inattendues permettent de conserver un personnel qualifié en attendant des temps meilleurs. Une petite usine d'électronique de la région de Nagoya spécialisée dans le travail de précision s'est mise à produire de minuscules tartelettes au chocolat garnies de fleurs séchées comestibles, innovation qui semble remporter un vif succès.

\section{Un écart qui se creuse entre les détenteurs d'un emploi permanent et les autres}

Cette crise révèle l'absence de véritable filet de protection pour un grand nombre de travailleurs au Japon. Selon des statistiques de l'OIT datant de mars, 77 \% des chômeurs ne disposent pas d'assurance de chômage, taux le plus bas parmi les pays développés.Un dessin très suggestif dans le grand quotidien Asahi daté du 8 février 2009 montre jusqu'où la chute peut s'effectuer si l'on ne dispose pas de protection sociale. En d'autres termes il met en évidence le fait que les intérims actuels sont de futurs sans abris. L'intérim s'est beaucoup développé ces dernières années jusqu'à atteindre $7 \%$ de la population active, $\mathrm{du}$ fait de son autorisation dans les emplois manufacturiers. La reconversion des contrats de sous-traitance en contrat d'intérim suite à plusieurs lois qui l'ont libéralisé n'a fait qu'accentuer ce phénomène. La mise à disposition par la sous-traitance de personnels qui part chez le donneur d'ordre a pour corollaire leur précarité, du fait de cette nouvelle mobilité. L'octroi d'un logement sommaire associé au travail offert entraîne systématiquement avec la perte de l'emploi, celle de ce dernier. Se retrouvant brutalement à la rue ce sont ces travailleurs qui manifestent et viennent grossir les rangs des sans abris dans les métropoles du Japon.

La main d'œuvre d'origine étrangère fait également la première les frais de ces politiques de réduction et on ne compte pas les émissions télévisées montrant des familles de sudaméricains principalement d'origine brésilienne repartant chez elles. Ces immigrants issus des générations de japonais parties au début $\mathrm{du} \mathrm{XX}^{\mathrm{e}}$ siècle chercher une vie meilleure dans l'hémisphère sud du continent américain ont bénéficié ces dernières décennies de toutes sortes d'avantages pour venir subvenir au manque crucial de population jeune dans un archipel en voie très rapide de vieillissement. Dans les grandes régions industrielles comme autour de Nagoya des quartiers entiers se sont mis à parler portugais ou espagnol. Les enfants d'âge scolaire ont fait découvrir au ministère japonais de l'éducation des problèmes jusque là inconnus dans l'archipel, comme l'insertion d'enfants ne parlant pas la langue et ayant un comportement peu conforme à l'attente habituelle. Cette population qui pensait s'installer au Japon pour de très longues années et avait fait le choix d'intégrer ses enfants dans la société d'accueil, se retrouve face à l'impossibilité de rester plus longtemps sur place. 
5 Les stagiaires étrangers, principalement originaires d'Asie du sud-est, qui finissaient par constituer une main d'œuvre économique pour nombre de PME, représentent les autres victimes immédiates de cette crise sans précédent venue frapper l'archipel. Bref ceux sont les plus vulnérables, sans oublier les handicapés, les prisonniers, qui constituaient une main d'œuvre docile et peu coûteuse pour l'industrie, qui sont les premiers touchés par ces mesures.

6 Mais les effets de la crise n'en restent pas là puisqu'ils frappent désormais au cœur même de la population. Les personnes âgées en font aussi les frais comme le démontrait un remarquable documentaire de la NHK en avril sur les maisons de retraite privées en quasi faillite, suite à la crise financière. Du fait de la revente successive de ces établissements entre des sociétés immobilières qui ont perdu tous leurs avoirs avec la crise, nombre d'entre elles se trouvent incapables d'assurer les services indispensables à l'entretien des lieux et au bien-être des pensionnaires. Face à des consultations de santé réduites à la portion congrue, des réparations de bâtiments ajournées etc. les pensionnaires qui ont vendu leur logement pour pouvoir payer le droit d'entrée, et les mensualités élevées de ce genre d'établissements se trouvent pratiquement sans recours.

7 Bref la société japonaise qui depuis l'après-guerre et jusqu'aux années 1980 se présentait comme une immense classe moyenne, montre de sérieux signes de division entre ceux qui bénéficient d'un emploi permanent et le reste de la population. Cet écartement est à l'œuvre depuis plusieurs années et la crise ne fait qu'accélérer ce phénomène. Elle témoigne également du faible sentiment de solidarité entre ses différentes composantes. Un ouvrage récent d'une journaliste japonaise résidant à Paris Yamamoto Miharu, intitulé France Révolte d'une jeunesse met l'accent sur ce qui différencie profondément ces deux sociétés. Prenant comme point de départ les manifestations de 2006 contre le CPE, elle explique que la jeunesse française peut se rebeller tout en ayant le soutien du monde des adultes, car il existe en France une très forte conscience des droits et un sentiment de solidarité. A l'opposé au Japon les licenciements qui se succèdent depuis le début de l'année n'ont provoqué aucune grande manifestation (seuls de très petits groupes d'intérims devenus sans abris ont osé rompre un peu le calme social). Cette absence de réactions serait à mettre au compte d'une étrangeté, caractérisée par une très grande froideur de la population vis à vis des autres, d'un manque de maturité du monde des adultes. Il ne faut pas oublier qu'élever la voix dans la société japonaise reste largement jugé comme asocial et cause d'embarras. En d'autres termes marquer de l'indifférence au sort de son voisin ne se perçoit pas du tout de la même façon dans les deux sociétés.

Quelques initiatives individuelles existent tout de même pour palier au manque de protection sociale, comme ce prêtre d'un petit monastère bouddhique dans le département de Miyazaki qui abrite des intérims licenciés dépourvus de toit et qui parvient à subvenir à leurs besoins grâce à l'aide du voisinage (Asahi, 7 février 2009). La crise financière contribue à mettre en lumière une société qui éprouve toujours du mal à remettre en question ses propres fondements et surtout à faire siennes des problématiques communes à la planète. Malgré des prises de conscience individuelle qui ne cessent de se multiplier (citons entre autres Amamiya Karin et son réseau contre la pauvreté), le Japon continue toujours à se percevoir globalement en vase clos, peu solidaire du reste du monde, à l'abri des périls extérieurs grâce à la présence des océans. L'usage toujours très répandu de l'expression Ware ware wa Nihonjin (Nous les japonais) par opposition au reste du monde en dit long sur ce sentiment. 


\section{Une floraison des cerisiers qui domine toujours les esprits}

Les émissions spéciales des chaînes de télévision ne montrent pas seulement la détresse de ceux qui ont tout perdu mais aussi les difficultés de ceux qui ont conservé leur emploi et doivent faire face à des temps incertains. Selon diverses statistiques les japonais auraient désormais tendance à économiser en emportant de plus en plus un repas froid sur leur lieu de travail, plutôt que de recourir à un repas rapide dans un établissement quelconque. Le bento, véritable institution culturelle japonaise qui a d'ailleurs commencé il y a quelques années son internationalisation, joue d'un regain de faveur chez la population masculine et la grande question est de savoir quels sont ses mets préférés.

10 En avril outre la candidature aux jeux olympiques d'été de la mairie de Tokyo et les sommes colossales en jeu pour leurs préparations, le thème de prédilection était la floraison des cerisiers. L'avancement de l'ouverture des boutons de fleurs à travers tout l'archipel a envahi brutalement l'actualité, reléguant la crise financière au second plan. La dégradation du savoir vivre des japonais occupait souvent la une des nouvelles. Tel prêtre d'un grand sanctuaire shintoïste urbain déplorait le manque de civisme de ses compatriotes, qui n'hésitaient pas à déposer leurs sacs d'ordure sur ceux du sanctuaire et à allumer des feux destinés à des barbecues sous les cerisiers, malgré l'interdiction. Leur chaleur aurait fait déjà périr de nombreux arbres! Certains des visiteurs n'hésiteraient pas à faire leur vaisselle avec l'eau destinée à la purification !

11 Si les produits de consommation destinés à des budgets limités se multiplient et les clients deviennent beaucoup plus attentifs aux différences de prix, la crise financière n'a pas encore modifié les modes de vie de façon profonde. Son apparition n'a fait qu'accélérer la recomposition de la grande distribution, en particulier la fusion des grands magasins, et renforcer la lutte acharnée livrée entre les grandes surfaces de type supermarchés et les kombini (superette de voisinage qui fonctionnent $24 \mathrm{~h}$ sur 24). Dans les centres villes des grandes métropoles le Japon montre toujours une extraordinaire richesse et une abondance de biens et de services assez exceptionnelle. Le luxe devient de plus en plus ostentatoire dans une société qui pendant des décennies restait très discrète dans ce domaine. L'écart entre ceux qui y ont accès et le reste de la population se creuse progressivement sans pour l'instant soulever de véritables questionnements.

12 Mais un autre péril venu de l'extérieur commence à s'abattre sur l'archipel, celui de la grippe $\mathrm{A}$ à l'annonce duquel le Japon a dore et déjà pris des mesures draconiennes. Les japonais sont invités à annuler tout voyage touristique à l'étranger et doivent suivre une procédure administrative contraignante si ils sont contraints à quitter l'archipel pour des raisons professionnelles. La crise économique actuelle ne va t'elle pas accélérer la prise de conscience au sein de la population japonaise de la très grande dépendance du Japon vis à vis de l'étranger, et peut-être accroître le sentiment de solidarité, car fondamentalement c'est la bonne santé des marchés extérieurs qui va conditionner la reprise de l'activité.

Le Japon dans la crise actuelle (Le 13 avril 2009)

Jean-Robert Pitte 
La crise économique mondiale affecte le Japon largement autant que les grandes puissances anciennement industrialisées d'Europe ou d'Amérique du nord. M. Kaoru Yosano, ministre de la Politique économique et budgétaire évoque un recul du PIB de $3,3 \%$ au dernier trimestre de 2008, par rapport au précédent. Les experts de l'OCDE prédisent un recul en 2009 de $6,6 \%$, contre $4,4 \%$ aux Etats-Unis. Les exportations reculent et la balance commerciale est déficitaire depuis novembre 2008.1300 entreprises ont fait faillite en février 2009. Le chômage est rapidement en train de passer de 4 à $5 \%$, chiffre jamais atteint depuis longtemps au Japon. Enfin, l'État peine à conduire la relance, car la dette publique est immense : $180 \%$ du PIB. Faut-il dès lors penser que la deuxième puissance du monde est définitivement à genoux ?

Comme lors de l'éclatement de la bulle à la fin des années 1990, les commentateurs occidentaux observent ces faits non sans une certaine satisfaction, tant la réussite de l'économie nipponne est longtemps apparue comme incompréhensible, voire insolente pour un pays ayant perdu la dernière guerre et qui, comme l'Allemagne, s'est relevé en un temps record. Dans les années 1997-1998, on pouvait lire à la une des grands journaux et magazines français : "fin du miracle », «illusions perdues », «le Japon à terre », etc. Et puis, sans que les Cassandre ne prennent la peine de battre leur coulpe, le Japon s'est relevé est a retrouvé croissance économique et prospérité. Cela ne s'est pas fait sans effort et cela ne veut pas dire que tous les problèmes aient été résolus et, parmi ceux-ci, le vieillissement de la population. D'ailleurs, de ce point de vue, la Chine s'avance inexorablement vers des lendemains qui déchantent, du fait de sa politique de l'enfant unique. L'allongement rapide de la durée de vie correspond au moment où arrivent en activité des classes d'âge très creuses.

Les causes de la crise sont évidemment les mêmes qu'ailleurs dans le monde et les liens étroits qui existent entre l'économie japonaise et l'économie américaine prennent ici toute leur importance. Certes, toutes les banques japonaises ne sont pas en bonne santé, mais les plus fragiles avaient été emportées lors de la crise précédente. Par ailleurs, si l'État japonais est lourdement endetté, ce n'est pas le cas des ménages qui ont conservé, même si c'est un peu à la baisse, leurs traditionnelles habitudes d'épargne. Les exportations souffrent d'une surévaluation du yen. Si le gouvernement japonais parvient à faire baisser ce dernier, il est certain qu'elles reprendront fortement, d'autant que les grandes entreprises sont capables d'adaptations rapides. Par ailleurs, n'oublions pas que le principal client du Japon est la Chine et que, même en perte de vitesse, les perspectives de croissance de ce puissant voisin demeurent très positives. Alors que le Monde du 8 avril 2009 titrait «En 2009, le Japon connaîtra la pire récession des pays industrialisés », M. Shogo Maeda, responsable des actions japonaises de la Compagnie financière Schroders Investments Limited à Londres déclarait en décembre 2008: «Globalement, nous estimons que le Japon est bien placé pour connaître une solide reprise une fois ce ralentissement cyclique achevé». L'exemple de Nissan est parlant: les ventes d'automobiles de la compagnie ont baissé de 5,9\% au Japon en 2008, mais elles ont augmenté de 5,5\% en Europe, 10,9 \% aux Etats-Unis et 19,1 \% en Chine !

Avant de prédire un avenir définitivement sombre au Japon, il faut songer aux remarquables capacités de ressaisissement et de rebondissement de sa population lors des moments les plus critiques de son histoire. Ses traditions sociales sont héritées d'une histoire sans invasions depuis deux millénaires. Ce peuple a fait preuve au fil des siècles d'une capacité rare à s'inspirer d'apports extérieurs, à adapter finement les idées d'autrui et d'ailleurs. Un bon exemple est celui de la philosophie chinoise de Confucius qui invite à l'effort individuel et collectif, au dépassement plus qu'à la résignation à laquelle on le| 
réduit souvent en Occident, à la recherche passionnée de la paix intérieure par les oeuvres. Dans ce pays, la justice est un bien trop précieux pour être un dû. Il tient sa forte identité de son insularité, mais aussi des deux siècles et demi de fermeture quasiintégrale voulue par la dynastie des shoguns Tokugawa (1603-1868) fondée par le visionnaire Ieyasu. Pendant cette longue période, l'État a forgé l'unité nationale et une architecture sociale stable, développé le sens de l'honneur, l'éducation pour tous, y compris les femmes qui avaient accès à l'écriture et savaient donc transmettre à leurs enfants, Les connaissances occidentales parvenaient, via les Hollandais, par le port de Nagasaki et furent ainsi assimilées au rythme choisi par les Japonais. C'est pourquoi l'ouverture des années 1860, puis la révolution Meiji de 1868 ne provoqua aucun traumatisme irréversible: les Japonais ont su profiter des techniques et des idées occidentales sans rien perdre de leur identité profonde. C'est pourquoi la dialectique entre tradition et modernité a moins de sens dans ce pays que partout ailleurs. La destruction de sa capitale, Tokyo, par le grand séisme de 1923 est une épreuve terrible dont le pays se relève vite, tant il a appris à maîtriser un environnement multi-insulaire, montagneux et morcelé, marqué par la violence des typhons, des tremblements de terre, des tsunamis, des inondations, des glissements de terrain, etc.. La deuxième guerre mondiale et sa fin terrifiante dans le feu atomique laissent le pays en ruines matérielles, mais non morales. Grâce à son énergie et à l'aide américaine, il devient très la deuxième puissance mondiale.

Longtemps, sa faiblesse fut sa difficulté à se faire comprendre du plus grand nombre, à parler de lui-même. Cet enfermement appartient au passé. En ce début du XXI siècle, la culture japonaise n'est plus confidentielle : elle est enfin sortie de ses frontières et touche tous les milieux. La planète entière a emprunté au Japon sa technologie électronique (photographie, son), son art graphique et cinématographique, sa gastronomie, sa mode vestimentaire, son architecture et son design, ses arts martiaux, etc. Seule la classe politique japonaise demeure discrète, voire inaudible: le contraste est saisissant avec l'omniprésence médiatique des chefs d'État ou de gouvernement du G8 ou du G20, mais est-ce vraiment un handicap? Le Japon sait qu'il a besoin du monde, mais le monde a compris que le Japon lui était nécessaire pour prospérer en paix.

15 Jean-Robert Pitte (jean-robert.pitte@wanadoo.fr) est professeur à l'Université de ParisSorbonne. Il est également Président de la Société de Géographie depuis le $1^{\mathrm{er}}$ janvier 2009, Président de l'Association pour le Développement du Festival International de Géographie de Saint-Dié-des-Vosges depuis 2008, Président de la Mission du Patrimoine et des Cultures Alimentaires (chargée par le Président de la République du classement de la gastronomie française au patrimoine immatériel de l'UNESCO). Il a publié notamment :

- (direction) : La Sorbonne. 750 ans au service des humanités, Paris, PUPS, 2007,

- Le désir du vin à la conquête du monde, Paris, Fayard, 2009.

- À la table des dieux, Paris, Fayard, 2009. 


\section{BIBLIOGRAPHIE}

Yamamoto Miharu, 2008, Furansu Junesu no hanran (France Révolte d'une jeunesse), Tokyo, Otsuki shoten.

\section{RÉSUMÉS}

La crise financière au Japon a frappé brutalement les industries exportatrices qui soutenaient depuis des décennies l'essor économique. Elle a révélé l'ampleur du fossé qui sépare désormais les travailleurs temporaires, premières victimes de cette crise, des employés permanents, amplifié par la faiblesse de la protection sociale. Cet écart qui ne cesse de s'accroître n'a cependant provoqué aucun mouvement social notable.

The financial crisis has severely hit all the export industries, which sustain the economic growth those last years. The gap between the temporary workers first victims of the crisis, and the fully employed ones, was revealed, and is enlarged by the weakness of the social protection. In spite of this gap, no social upheaval can be noticed.

\section{INDEX}

Mots-clés : Japon, crise financière, intérim, protection sociale, solidarité

Keywords : Japan, financial crisis, temporary worker, social protection, solidarity

\section{AUTEUR}

\section{SYLVIE GUICHARD-ANGUIS}

Sylvie Guichard-Anguis (sguichard_anguis@hotmail.com) est chargé de recherche au CNRS et est accueillie par Laboratoire Espaces, Nature et Culture (CNRS/Paris-Sorbonne Paris 4). Elle a publié récemment :

- Guichard-Anguis Sylvie and Moon Okpyo (ed.), 2008, Japanese Tourism and the Culture of Travel, London and New York, Routledge, $221 \mathrm{p}$.

- Sylvie Guichard-Anguis et Stéphane Héritier (dir.), 2008, Patrimoine naturel : entre culture et ressource, Géographie et Cultures $\mathrm{n}^{\circ} 66$ automne 2008, $143 \mathrm{p}$.

- Guichard-Anguis Sylvie, 2009, "Une divine obésité : les sumotori au Japon", Csergo Julia (dir.), Trop gros ? L'obésité et ses représentations, Editions Autrement (coll. Mutations), p.160-173 